\title{
Hypothermic Oxygenated Machine Perfusion of Extended Criteria Kidney Allografts from Brain Dead Donors: Protocol for a Prospective Pilot Study
}

Franziska Alexandra Meister ${ }^{1}$, MD; Zoltan Czigany ${ }^{1}$, MD; Jan Bednarsch ${ }^{1}$, MD; Jörg Böcker ${ }^{1}$, MD; Iakovos Amygdalos ${ }^{1}, \mathrm{MD}$; Daniel Antonio Morales Santana ${ }^{1}$, MD; Katharina Rietzler ${ }^{1}$; Marcus Moeller ${ }^{2}$, Prof Dr; René Tolba $^{3}$, Prof Dr; Peter Boor ${ }^{4}$, Prof Dr; Wilko Rohlfs ${ }^{5}$, Dr-Ing; Ulf Peter Neumann ${ }^{1}$, Prof Dr; Georg Lurje ${ }^{1}$, MD

${ }^{1}$ Department of Surgery and Transplantation, University Hospital, Rheinisch-Westfälische Technische Hochschule Aachen, Aachen, Germany

${ }^{2}$ Division of Nephrology, Department of Medicine II, University Hospital, Rheinisch-Westfälische Technische Hochschule Aachen, Aachen, Germany

${ }^{3}$ Institute for Laboratory Animal Science and Experimental Surgery, University Hospital, Rheinisch-Westfälische Technische Hochschule Aachen, Aachen, Germany

${ }^{4}$ Institute of Pathology, University Hospital, Rheinisch-Westfälische Technische Hochschule Aachen, Aachen, Germany

${ }^{5}$ Institute of Heat and Mass Transfer, Rheinisch-Westfälische Technische Hochschule Aachen, Aachen, Germany

\section{Corresponding Author:}

Georg Lurje, MD

Department of Surgery and Transplantation

University Hospital, Rheinisch-Westfälische Technische Hochschule Aachen

Pauwelsstr 30

52074 Aachen

Germany

Phone: 492418089501

Email: glurje@ukaachen.de

\section{Abstract}

Background: Kidney transplantation is the only curative treatment option for end-stage renal disease. The unavailability of adequate organs for transplantation has resulted in a substantial organ shortage. As such, kidney donor allografts that would have previously been deemed unsuitable for transplantation have become an essential organ pool of extended criteria donor allografts that are now routinely being transplanted on a global scale. However, these extended criteria donor allografts are associated with significant graft-related complications. As a result, hypothermic oxygenated machine perfusion (HOPE) has emerged as a powerful, novel technique in organ preservation, and it has recently been tested in preclinical trials in kidney transplantation. In addition, HOPE has already provided promising results in a few clinical series of liver transplantations where the liver was donated after cardiac death.

Objective: The present trial is an investigator-initiated prospective pilot study on the effects of HOPE on extended criteria donor allografts donated after brain death and used in kidney transplantation.

Methods: A total of 15 kidney allografts with defined inclusion/exclusion criteria will be submitted to two hours of HOPE via the renal artery before implantation, and are going to be compared to a case-matched group of 30 patients (1:2 matching) who had kidneys transplanted after conventional cold storage. Primary (posttransplant dialysis within 7 days) and secondary (postoperative complications, early graft function, duration of hospital and intensive care unit stay, and six-month graft survival) endpoints will be analyzed within a six-month follow-up period. The extent of ischemia-reperfusion injury will be assessed using kidney tissue, perfusate, and serum samples taken during the perioperative phase of kidney transplantation

Results: The results of this trial are expected in the first quarter of 2020 and will be presented at national and international scientific meetings and published in international peer-reviewed medical journals. The trial was funded in the third quarter of 2017 and patient enrollment is currently ongoing.

Conclusions: This prospective study is designed to explore the effects of HOPE on extended criteria donor kidney allografts donated after brain death. The present report represents the preresults phase.

Trial Registration: Clinicaltrials.gov NCT03378817; https://clinicaltrials.gov/ct2/show/NCT03378817 
(JMIR Res Protoc 2019;8(10):e14622) doi: 10.2196/14622

\section{KEYWORDS}

hypothermic oxygenated machine perfusion; donation after brain death; extended criteria donor; kidney transplantation; kidney transplant; organ donation

\section{Introduction}

Since Joseph Murray’s pioneering efforts in 1954, allogenic kidney transplantation has evolved as the standard treatment for end-stage renal disease [1]. In 2018, approximately 8000 patients were waiting for kidney transplant in Germany, but only 2005 transplant procedures using postmortem organs were performed due to organ scarcity [2]. Several strategies for donor pool expansion are being pursued concurrently. These include the use of old donors, living donors, and extended criteria donor (ECD) allografts for kidney transplant. However, ECD-allografts exhibit poor tolerance to ischemia-reperfusion injury (IRI), an important cause of kidney damage [3,4]. As such, IRI is usually the underlying cause of graft dysfunction in ECD kidney transplant [5]. Primary graft nonfunction, leading to graft loss and retransplantation, as well as delayed graft function (DGF) with the need for posttransplant dialysis are the most frequent clinical manifestations of this pathology [5].

Dynamic organ preservation approaches, such as machine perfusion, hold promise for improving organ preservation and resuscitation of marginal donor allografts [6-9]. Although tissue oxygen consumption is markedly decreased at $4-10^{\circ} \mathrm{C}$, there is still relevant metabolism at this temperature. Oxygen during machine perfusion is the key ingredient in the protective responses of hypothermic oxygenated machine perfusion (HOPE), with organ reconditioning effectively increasing the cellular energy balance via various mitochondrial pathways [10]. Over time, the addition of oxygen during machine perfusion emerged as a powerful novel tool in organ preservation $[11,12]$. HOPE is an easy to implement, short-term, end-ischemic reconditioning concept, as the organ perfusion is performed in the transplant center shortly before the actual implantation $[11,13]$. Ischemia-reperfusion injury occurs upon revascularization of the kidney allograft. Decrease of oxidative metabolism and depletion of adenosine triphosphate (ATP) are the results of the lack of oxygen during ischemia. Reintroduction of oxygen to the ischemic allograft results in massive reactive oxygen species production and release, leading to cellular damage. The mechanisms behind IRI are multifactorial, and ways to minimize its detrimental effects are still the subject of intense debate [14].

In the preclinical setting, the positive effects of HOPE have recently been demonstrated through a reduction in the incidence of tubular damage, macrophage activation, and functional optimization of cellular energy-status in vitro and in vivo $[8,9,11]$. There are several hypotheses regarding the underlying mechanisms of HOPE-induced organ protection. According to these hypotheses, HOPE presumably exerts its positive effects by: (1) modulating cellular metabolism (energy balance, mitochondrial respiration, IRI); (2) stimulating the vascular endothelial layer; and (3) triggering various subcellular protective pathways (Figure 1) [15]. Investigating the effects of HOPE in a rat model, Kron et al recently demonstrated its beneficial effects on the immune response. Less cytokine release and less T-cell and macrophage activation were measured in the animals receiving the HOPE-treated kidneys [16]. Accordingly, decreased immune response may be one of the key underlying mechanisms of HOPE.

The aim of this prospective cohort study is to investigate the effects of HOPE on ECD kidney allografts donated after brain death. This study utilizes a comprehensive sample collection protocol to assess the subcellular mechanisms of HOPE in the clinical setting. 
Figure 1. Hypothermic oxygenated machine perfusion. The donor organ is explanted and transported to the transplant center stored in a box of ice. In the transplant center, the kidney is connected to the Kidney Transport Assist device and HOPE is performed. It is assumed HOPE leads to less oxidative stress, decreased cell death and enhanced energy reserves. Adapted from Czigany et al [\&lt;xref ref-type="bibr" rid="ref12"\&gt;12\&lt;/xref\&gt;]. ET: Eurotransplant, HOPE: hypothermic oxygenated machine perfusion; ATP: adenosine triphosphate; ROS: reactive oxygen species.

In a Peripheral Hospital within the ET network

Organ Donation and Transport to the Transplant Center
In the Transplant Center

\section{Back Table HOPE vs. Conventional Cold Storage}

HOPE, Kidney Assist Device
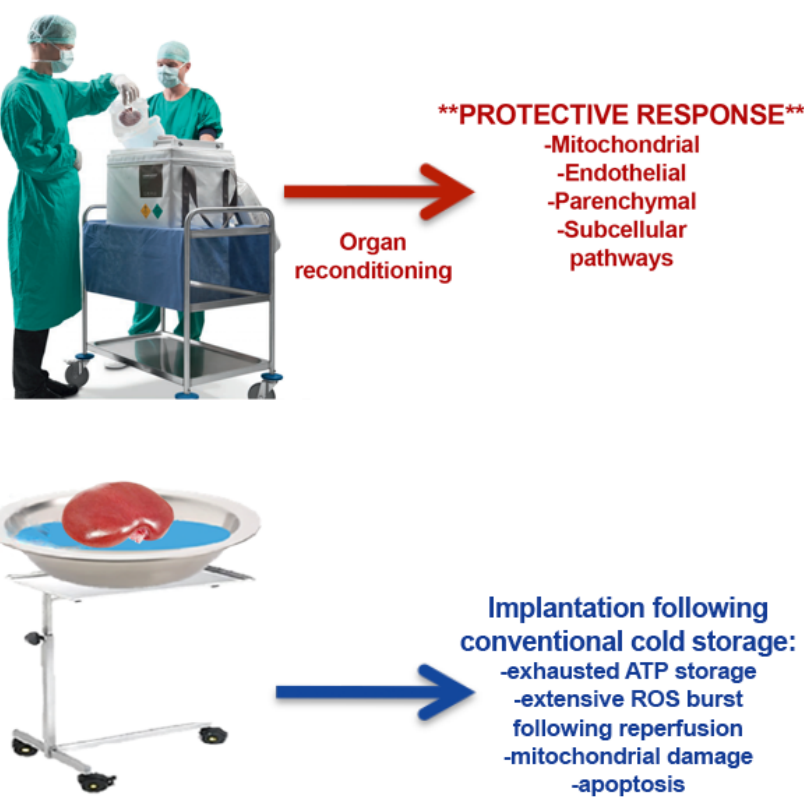

Conventional cold storage

Patients older than 18 years of age, suffering from end-stage renal disease (ESRD), listed for kidney transplant, and receiving ECD organs at the Department of Surgery and Transplantation, University Hospital Rheinisch-Westfälische Technische Hochschule (RWTH) Aachen, Aachen, Germany, are eligible for the study. Informed consent was obtained from all subjects participating in the trial by a qualified member of the study team. Inclusion and exclusion criteria are listed in Textbox 1. 
Figure 2. Study flow chart. ECD: extended criteria donor; HOPE: hypothermic oxygenated machine perfusion; DGF: delayed graft function; GFR: glomerular filtration rate.

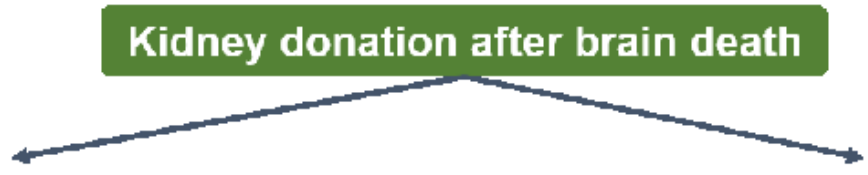

\section{Inclusion criteria}

1. Signed informed consent

2. Patients 18 years or older

3. Patients suffering from end stage kidney disease

4. Listed for kidney transplantation

5. Receiving ECD-allografts

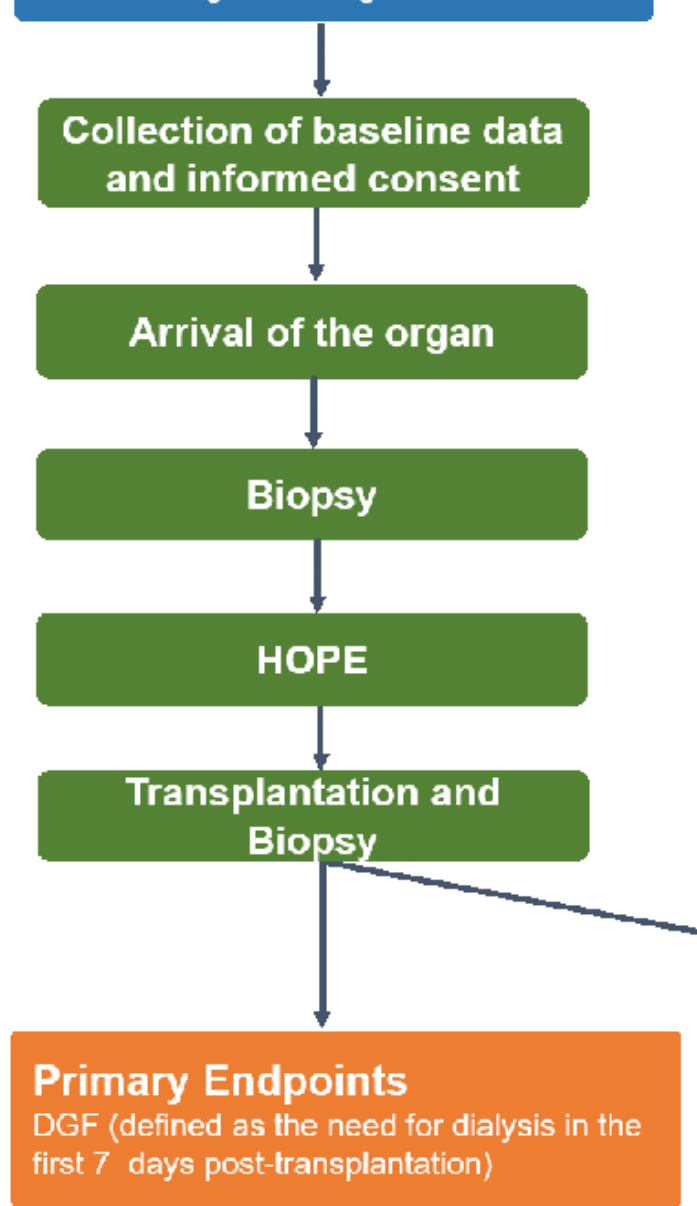

\section{Exclusion criteria}

1. Recipients of living donor kidney transplants

2. Previous kidney transplantation

3. Participation in other kidney-related trials

4. The subject received an investigational drug within 30 days prior to inclusion

5. The subject is unwilling or unable to follow the procedures outlined in the protocol

6. The subject is mentally or legally incapacitated

7. Non-German or non-English speakers

8. Family members of the investigators or employees of the participating department

9. The subject is mentally or legally incapacitated

10. The subject suffers from uncontrolled bacterial or viral infection

\section{Secondary Endpoints}

1. Incidence of pastoperative complications as assessed by the Clavien-Dindo complication score and the comprehensive complication index

2. Further laboratory parameters such as GFR, creatinine reduction ratio, hemoglobin

3. Duration of intensive care stay

4. Duration of hospital stay

5. Biomarkers of ischemia-reperfusion injury and inflammation 
Textbox 1. Study inclusion and exclusion criteria.

\author{
Inclusion Criteria: \\ - $\quad$ Signed informed consent \\ - 18 years or older \\ - $\quad$ Suffering from ESRD (end-stage renal disease) \\ - $\quad$ Listed for kidney transplant \\ - Receiving ECD (extended criteria donor)-allografts \\ Exclusion criteria: \\ - Recipients of living donor kidney transplants \\ - Previous solid organ transplantation \\ - Combined procedures (eg, kidney-liver transplantation) \\ - Participation in other kidney-related trials \\ - The subject received an investigational drug within 30 days prior to inclusion \\ - The subject is unwilling or unable to follow the procedures outlined in the protocol \\ - The subject is mentally or legally incapacitated \\ - $\quad$ Non-German or non-English speakers \\ - Family members of the investigators or employees of the participating department \\ - The subject suffers from uncontrolled bacterial or viral infection
}

\section{Organ Procurement and Definition of Extended Criteria Donor Criteria}

ECD kidney allografts will be retrieved by local procurement teams with the Eurotransplant network. Following cross-clamping (in situ flushing of the abdominal organs and the beginning of cold ischemia time), allografts will be removed and transported in a standardized fashion on packed ice. Required data regarding the donor and the organ will be collected and will be transferred to designated case report files (CRFs).

As per previous studies [4,17] deceased donors must be $\geq 60$ years old, but it is possible for those aged between 50-59 years old to be donors if they have at least two of the following conditions: (1) cerebrovascular cause of death; (2) serum creatinine greater than $1.5 \mathrm{mg} / \mathrm{dL}(132.6 \mu \mathrm{mol} / \mathrm{L})$; or $(3)$ history of arterial hypertension.

\section{Hypothermic Oxygenated Machine Perfusion Versus Historic Cold Storage Group}

Primary and secondary outcomes of patients transplanted with the HOPE-treated kidneys will be compared to a case-matched group of 30 patients (1:2 matching) transplanted with ECD organs after conventional cold storage, using identical surgical techniques and perioperative treatment at the Department of Surgery and Transplantation, University Hospital RWTH Aachen. The present trial comprises two groups, a perfusion (Group 1, HOPE) and a control conventional cold storage group (Group 2, Historic). Patients on the waiting list for kidney transplant with proven written consent will be recruited. HOPE will be applied to the allograft in the operating room after regular organ procurement, transport, and back-table preparation (Figure 1).

We adapted a protocol based on clinical studies with nonoxygenated hypothermic machine perfusion, and on preclinical experience with HOPE $[11,18,19]$. HOPE will be applied through the renal artery for at least two hours, with a perfusion pressure of $25 \mathrm{mmHg}$ and a perfusion rate of $50-200 \mathrm{ml} / \mathrm{min}$ using $1 \mathrm{~L}$ of recirculated perfusate at $0-4^{\circ} \mathrm{C}$. The perfusate will be oxygenated using medical grade oxygen $\left(\mathrm{O}^{2}\right)$, with a partial pressure of oxygen $\left(\mathrm{pO}^{2}\right)$ of 60-80 kilopascals $(\mathrm{kPa})$, by an oxygenator included as a disposable part of the setup. Perfusion parameters registered by the device will be stored automatically and evaluated for possible patterns. Immediately prior to perfusion, grafts will be flushed with perfusate solution to wash out the residual histidine-tryptophan-ketoglutarate (HTK) solution. Storage, management, and use of the medical products will be carried out according to the manufacturer's guidelines.

\section{Matching Criteria}

Donor urine output over the last 24 hours before retrieval, cold ischemic time (CIT), and the recipients Charlson comorbidity index [20] have been selected as matching criteria. Matching will be performed using a propensity score [21].

\section{Kidney Transplantation}

Kidney grafts will be implanted heterotopically to the iliac fossa, as per our institutional protocol. The renal vein is anastomosed first, end-to-side to the external iliac vein, followed by renal artery anastomosis to the external iliac artery in the same fashion. After reperfusion of the kidney, the ureter is implanted 
into the bladder, according to Lich-Gregoir, and the uretero-cystostomy is stented with a 6-French double-J stent.

\section{Sample Collection and Storage}

Ischemia reperfusion injury will be assessed using kidney tissue samples taken upon arrival of the organ (before HOPE) and at the end of implantation after reperfusion, but before closure of the abdomen, to evaluate the extent of IRI (Figure 3). In total, two biopsies using BARD MONOPTY Disposable Core Biopsy Instrument (Bard Biopsy Systems Inc, Tempe, Arizona) will be harvested from the ECD kidney allograft and used for translational research. During machine perfusion, perfusate samples will be collected every 20 minutes. Blood samples are taken as part of the daily routine during the perioperative and postoperative course of kidney transplant (Figure 3). Blood parameters of kidney function (ie, creatinine, urea, estimated glomerular filtration rate [eGFR]) will be monitored. An additional $20 \mathrm{~mL}$ of blood will be drawn on postoperative days 1, 2, 3 and 7 and will be used for translational research (Figure 3). All kidney tissue, serum, and perfusate will be directly snap-frozen in liquid nitrogen $\left(-80^{\circ} \mathrm{C}\right)$ and stored for 6 months after completion of the trial. To assess the subcellular mechanisms of HOPE in the clinical setting, various parameters, including markers of kidney injury (creatinine and neutrophil gelatinase-associated lipocalin (NGAL) in plasma samples), tissue ATP concentration, inflammatory mediators (eg, tumor necrosis factor alpha, interleukin-6, macrophage migration inhibitory factor, interleukin-10, monocyte chemoattractant protein-1), and biomarkers of oxidative damage (eg, 8-hydroxy-2'-deoxyguanosine), will be analyzed [11,12].

Figure 3. Interventions and study visits. Numbers represent the single study visit: Visit 1: screening, enrollment; Visit 2: admission; Visits 3, 4, and 5: postoperative days 1,2, and 3; Visit 6: seventh postoperative day; Visit 7: discharge; Visit 8: 6 months follow-up; final visit. HOPE: hypothermic oxygenated machine perfusion.
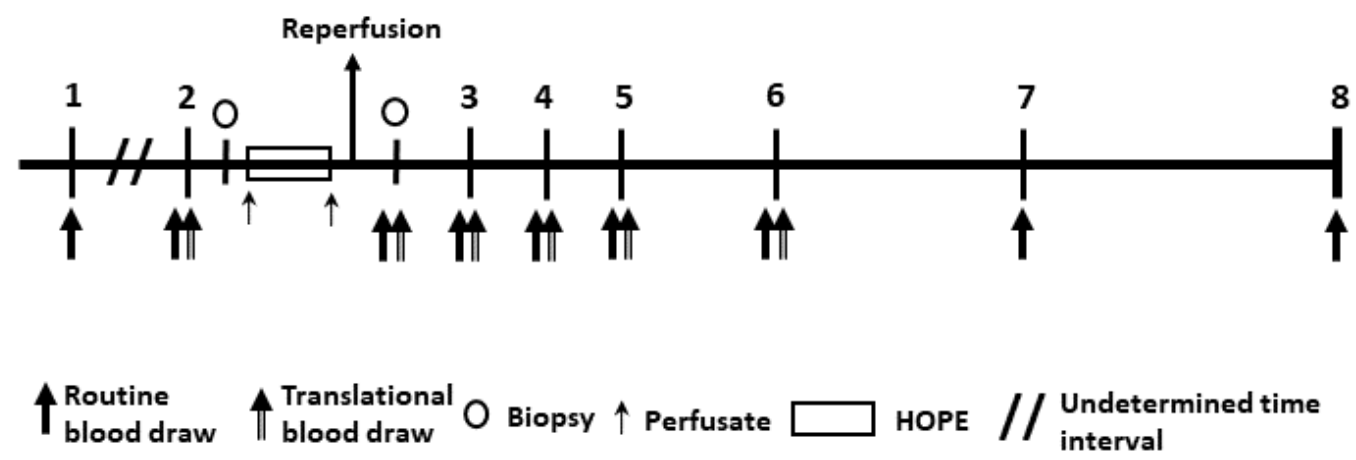

\section{Immunosuppression and Postoperative Care}

All patients will be treated according to our institutional protocol. Aside from HOPE for the donor kidneys, all procedures, including peri- and intraoperative management, are conducted in accordance with standard surgical kidney transplant management. The applied immunosuppressive regimen is based on intraoperative induction therapy with intravenous methylprednisolone and either basiliximab or thymoglobulin followed by corresponding oral doses of prednisolone, tacrolimus, and mycophenolate mofetil.

\section{Study Endpoints}

The primary endpoint is DGF, which is defined as the need for dialysis in the first 7-days posttransplantation. The secondary endpoints of the study include: (1) duration of DGF (defined as the period between kidney transplant and last dialysis in days) and functional DGF (defined as $<10 \%$ fall in serum creatinine for 3 consecutive days in the first week posttransplantation); (2) creatinine reduction ratio day 2 ([creatinine day 1 -creatinine day 2]/creatinine day 1) and Creatinine reduction ratio day 5 ([pretransplant creatinine-creatinine day 5]/pretransplant creatinine); (3) incidence of postoperative complications, as assessed by the Clavien-Dindo complication score and the comprehensive complication index [22]; (4) duration of intensive care and hospital stay; (5) recipient and graft survival after six months; (6) renal function (assessed by serum creatinine and eGFR) at one-, three- and six-months post-transplant; and (7) basic and translational research, which involves assessing biomarkers from routinely harvested kidney tissue and serum following implantation to obtain information on kidney injury, extent of oxidative damage, redox- and energetic homeostasis of the implanted donor kidney.

\section{Study Population}

To the best of our knowledge, aside from a sole case of HOPE-treated dual kidney transplant reported recently, no experience of HOPE in human donation after brain death kidney transplant has been published yet [23]. In the present pilot study, 15 patients $(\mathrm{N}=15)$ will be treated with HOPE to gain first prospective clinical knowledge. HOPE-treated patients will be compared to a case matched group of 30 patients $(\mathrm{N}=30)$.

\section{Data Collection and Statistics}

Patients will be given consent forms while in our outpatient clinic. Data will be obtained using study specific CRFs completed by the members of the study team. Subjects will be informed about data protection, including pseudonymization. 
Encoded data will only be provided to authorized persons (ie, authorized study staff, authorities, institutional review board). The study will be prematurely terminated for any individual subject in case of study-related complications or if the subject withdraws informed consent. Parametric and nonparametric tests will be done according to the normality of the data distribution. Two-way analysis of variance will be used for the time-course analysis of laboratory parameters. The log-rank test will be used for comparisons between Kaplan-Meier curves of six-month graft survival. Statistical significance will be defined as $P<.05$.

\section{Safety Considerations}

Exclusively certified medical products will be used. Blood sampling and tissue biopsies during the transplant procedure will be performed according to clinical standards, preventing any relevant study-related risks or additional burden on the subjects. An interim analysis will be performed as soon as 10 patients are HOPE-treated. The trial will be terminated immediately if the following criteria is fulfilled: significantly higher DGF rate $(P<.05$ using the Chi-squared test $)$ in the HOPE group compared to the matched conventional cold storage group (efficacy).

\section{Ethics}

The present trial will be carried out in compliance with the current version of the Declaration of Helsinki, good clinical practice guidelines (International Conference on Harmonization-Good Clinical Practice [ICH-GCP]), and all national legal and regulatory requirements. The institutional review board of the University RWTH Aachen has approved the study protocol, including consent form and patient information leaflet (EK 184/17). Members of the study team have completed a course in good clinical practice as certified by the German Medical Chamber.

\section{Study Group}

The study group of the present trial comprises the trial sponsor (GL, UPN) and the principal investigator (GL) of the University Hospital RWTH Aachen

\section{Ischemia-Reperfusion Injury and Inflammation}

IRI is an inevitable event in organ transplantation. Reduction of oxygen delivery because of blood flow interruption leads to anaerobic metabolism and the production of oxygen-free radicals and oxidative stress as a respiratory burst at the onset of reperfusion. IRI plays a key role in DGF and primary graft nonfunction following kidney transplantation, depending on initial organ quality $[8,9,24,25]$. To detect the effects of HOPE on IRI, the kidney tissue, blood, and perfusate samples obtained in this study will be used to quantify several innovative parameters of inflammation (eg, interleukins, tumor necrosis factor-alpha), kidney injury (Cytatin-C, NGAL, etc) energy(ATP levels) $[11,26]$, and redox-threshold (Hemoxygenase-1, Malondialdehyde) [12,27]. Luminometry, Spectrophotometry, Luminex-assay, Enzyme-linked immunosorbent assay (ELISA), Reverse transcriptase polymerase chain reaction (RT-PCR) and Western blot will be used for these analyses. Proteomics and metabolomics analysis will be performed on kidney tissue samples to potentially identify early mediators of HOPE-mediated organ protection.

\section{Results}

Results of this trial will be presented at national and international scientific meetings and published in international peer-reviewed medical journals. The trial was funded in the third quarter of 2017. Patient enrollment is currently ongoing, and the first results are expected in the first quarter of 2020.

\section{Discussion}

Allogenic kidney transplantation has evolved as the mainstay of treatment for end-stage renal disease $[3,28]$. The decreasing availability of quality organs for transplantation has resulted in substantial organ shortage, and as such kidney donor allografts that would have previously been deemed unsuitable for transplantation have become an essential organ pool of ECD-allografts that are now routinely being transplanted on a global scale. However, the use of ECD allografts is associated with IRI and a higher incidence of primary graft nonfunction or DGF [5]. The restoration of blood flow after cold and warm ischemia leads to paradox via massive release of reactive oxygen species, cytokines, chemokines, and activation of leukocytes [29]. Although machine perfusion in kidney transplantation was explored in the 1970's, it has not been widely used over the last few decades. Due to technical improvements and the addition of oxygen during machine perfusion, HOPE has evolved into a promising novel tool in organ preservation [11,12]. The superiority of HOPE over nonoxygenated cold machine perfusion and conventional cold storage was recently demonstrated in some preclinical experiments as well as in clinical trials using HOPE in donation after cardiac death liver transplantation [11,12,15]. A recent kidney transplantation rodent model in vivo study by Kron et al compared conventional cold storage, normothermic oxygenated blood perfusion, HOPE, and hypothermic deoxygenated perfusion using nitrogenated perfusate, and demonstrated superior effects of HOPE on macrophage activation, endothelium activation, tubular damage, and graft function as compared to other preservation methods [11].

We identified four active clinical trials exploring the effects of HOPE in kidney transplantation. In a pilot study by Ravaoili et al, 20 subjects were recruited to receive either HOPE-treated ECD-kidney or ECD-liver allografts. Whether the organs used were donated after cardiac death or donated after brain death remains unclear (NCT03031067; Table 1). One trial (NCT02621281) is a large multi-center RCT investigating HOPE in donated after cardiac death kidney transplantation, including ECD and nonECD allografts in a large Chinese cohort. Two relevant trials of the Consortium for Organ Preservation in Europe are recruiting in a large collective of patients. The Cold oxygenated machine preservation of aged renal donation after cardiovascular death transplants (COMPARE) trial (ISRCTN32967929), initiated at the University Hospital Leuven, Belgium, included a total of 102 transplanted donated after cardiac death kidney pairs that are being used to discover whether continuous oxygenated machine perfusion is superior 
to nonoxygenated machine perfusion. The COMPARE trial has a considerably different design compared to our study, with a logistically complex perfusion concept involving the transport of the machines to the retrieval center and applying continuous HOPE. Preimplantation oxygenated hypothermic machine perfusion reconditioning after cold storage (COPE-POMP) (ISRCTN63852508), initiated from the University Hospital of Essen, Germany, is currently ongoing and involves randomizing ECD allografts to end-ischemic preimplantation HOPE or to conventional cold storage.

Although we have designed our trial carefully, nonrandomization of the study groups and nonblinding of the transplant team, as well as matching with a historical group, are limitations of the present study. Nevertheless, conclusive clinical data about end-ischemic HOPE in human kidney transplantation are still missing and larger multi-center studies on this topic are currently underway. Therefore, it seems plausible to conduct this trial as a pilot study with a focus on short-term outcomes as well as translational research aspects of HOPE in ECD-kidney transplantation.

The present trial, nevertheless, possesses some specific strengths in that it focuses on patients solely receiving ECD-allografts, a population in which we anticipate the best cost/benefit ratio from the utilization of HOPE. Kidney transplant outcomes stem from a combination of various factors. Our matching criteria have been carefully selected based on previous clinical evidence to include both donor and recipient factors, which have a key role to play in posttransplant outcome [30,31]. Regarding translational research and sample collection, our study design as a single center prospective trial may also be beneficial. Samples will be obtained according to our study protocols and by the same study members throughout the whole study period. The results of the translational part of this study may deliver novel insights into the underlying subcellular effects of HOPE in human kidney transplantation.

Table 1. Active prospective clinical trials on HOPE (hypothermic oxygenated machine perfusion) in kidney transplants (as assessed on clinicaltrials.gov and isrctn.com on February 27, 2019).

\begin{tabular}{|c|c|c|c|c|c|}
\hline Trial number & Study center & Study type & Enrollment & Donor group & $\mathrm{MP}^{\mathrm{a}}$ point of time \\
\hline $\begin{array}{l}\text { NCT03378817 } \\
\text { (Present trial) }\end{array}$ & $\begin{array}{l}\text { University Hospital Aachen, } \\
\text { Aachen, Germany }\end{array}$ & $\mathrm{CS}^{\mathrm{b}}$ & 15 & $\begin{array}{l}\mathrm{ECD}^{\mathrm{c}} \text {-donated after } \\
\text { brain death }\end{array}$ & End-ischemic \\
\hline NCT03031067 & $\begin{array}{l}\text { University of Bologna, Bologna, } \\
\text { Italy }\end{array}$ & CS & 10 & $\begin{array}{l}\text { ECD (donated after } \\
\text { brain or cardiac death } \\
\text { unclear) }\end{array}$ & Unclear \\
\hline NCT02621281 & $\begin{array}{l}\text { First Affiliated Hospital Xi'an } \\
\text { Jiaotong University, Xi'an, } \\
\text { Shaanxi, China }\end{array}$ & $\mathrm{RCT}^{\mathrm{d}}$ & 400 & $\begin{array}{l}\text { donated after cardiac } \\
\text { death }\end{array}$ & $\begin{array}{l}\text { Immediately after re- } \\
\text { trieval }\end{array}$ \\
\hline ISRCTN32967929 & $\begin{array}{l}\text { University of Leuven, Leuven, } \\
\text { Belgium }\end{array}$ & RCT & 210 & $\begin{array}{l}\text { donated after cardiac } \\
\text { death }\end{array}$ & $\begin{array}{l}\text { Immediately after re- } \\
\text { trieval }\end{array}$ \\
\hline ISRCTN63852508 & $\begin{array}{l}\text { University Clinic Essen, Essen, } \\
\text { Germany }\end{array}$ & RCT & 262 & $\begin{array}{l}\text { ECD-donated after } \\
\text { brain death }\end{array}$ & End-ischemic \\
\hline
\end{tabular}

${ }^{\mathrm{a}}$ MP: machine perfusion.

${ }^{\mathrm{b}} \mathrm{CS}$ : cohort study.

${ }^{\mathrm{c}} \mathrm{ECD}$ : extended criteria donor.

${ }^{\mathrm{d}} \mathrm{RCT}$ : randomized controlled trial.

\section{Acknowledgments}

The authors have received funding from the START-Program of the Faculty of Medicine, RWTH Aachen (\#136/17 to GL and \#23/19 to ZC) and from the Excellence Initiative of the German federal and state governments (G:(DE-82) ZUK2-SF-OPSF443 to GL).

\section{Authors' Contributions}

The initial study concept was derived from the initiating investigator study group: GL, FAM, ZC, and UPN. FAM, GL, and ZC drafted the manuscript. JB, JoB, IA, KR, DAMS, MM, PB, WR, and UPN participated in designing the study, preparing the revised protocol, and functioning as local investigators at the University Hospital RWTH Aachen. All authors read and approved the final version of the manuscript.

\section{Conflicts of Interest}

None declared.

\section{References}


1. Watts G. Joseph Murray: innovative surgeon and pioneer of transplantation. The Lancet 2011 Mar;377(9770):987. [doi: $\underline{10.1016 / \mathrm{s} 0140-6736(11) 60375-9]}$

2. Eurotransplant. Eurotransplant. 2019. ET Yearly Statistics Overview 2018 URL: http://statistics.eurotransplant.org/index. php?search_type=overview\&search_text=9023 [accessed 2019-09-23]

3. Schaubel D, Desmeules M, Mao Y, Jeffery J, Fenton S. Survival experience among elderly end-stage renal disease patients. A controlled comparison of transplantation and dialysis. Transplantation 1995 Dec 27;60(12):1389-1394. [doi: 10.1097/00007890-199560120-00003] [Medline: 8545862]

4. Aubert O, Kamar N, Vernerey D, Viglietti D, Martinez F, Duong-Van-Huyen JP, et al. Long term outcomes of transplantation using kidneys from expanded criteria donors: prospective, population based cohort study. BMJ 2015 Jul 31;351:h3557 [FREE Full text] [doi: 10.1136/bmj.h3557] [Medline: 26232393]

5. Hameed AM, Pleass HC, Wong G, Hawthorne WJ. Maximizing kidneys for transplantation using machine perfusion. Medicine 2016;95(40):e5083. [doi: 10.1097/md.0000000000005083]

6. Moers C, Pirenne J, Paul A, Ploeg RJ. Machine Perfusion or Cold Storage in Deceased-Donor Kidney Transplantation. N Engl J Med 2012 Feb 23;366(8):770-771. [doi: 10.1056/nejmc1111038]

7. Jochmans I, Nicholson ML, Hosgood SA. Kidney perfusion: some like it hot others prefer to keep it cool. Current Opinion in Organ Transplantation 2017;22(3):260-266. [doi: 10.1097/mot.0000000000000405]

8. Tolba RH. Improved Preservation of Warm Ischemia-Damaged Porcine Kidneys after Cold Storage in Ecosol, a Novel Preservation Solution. Ann Transplant 2015;20:233-242. [doi: 10.12659/aot.892823]

9. Kalenski J, Mancina E, Paschenda P, Beckers C, Bleilevens C, Tóthová Ľ, et al. Comparison of Aerobic Preservation by Venous Systemic Oxygen Persufflation or Oxygenated Machine Perfusion of Warm-Ischemia-Damaged Porcine Kidneys. Eur Surg Res 2016 Apr 13;57(1-2):10-21. [doi: 10.1159/000444851] [Medline: 27070724]

10. Minor T, Scott WE, Rizzari MD, Suszynski TM, Luer B, Efferz P, et al. Energetic recovery in porcine grafts by minimally invasive liver oxygenation. J Surg Res 2012 Dec;178(2):e59-e63. [doi: 10.1016/j.jss.2012.01.018] [Medline: 22445459]

11. Kron, Schlegel A, de Rougemont O, Oberkofler CE, Clavien PA, Dutkowski P. Short, Cool, and Well Oxygenated - HOPE for Kidney Transplantation in a Rodent Model. Ann Surg 2016 Nov;264(5):815-822. [doi: 10.1097/SLA.0000000000001766] [Medline: 27584571]

12. Czigany Z, Schöning W, Ulmer TF, Bednarsch J, Amygdalos I, Cramer T, et al. Hypothermic oxygenated machine perfusion (HOPE) for orthotopic liver transplantation of human liver allografts from extended criteria donors (ECD) in donation after brain death (DBD): a prospective multicentre randomised controlled trial (HOPE ECD-DBD). BMJ Open 2017 Oct 10;7(10):e017558 [FREE Full text] [doi: 10.1136/bmjopen-2017-017558] [Medline: 29018070]

13. Dutkowski P, Schlegel A, de Oliveira M, Müllhaupt B, Neff F, Clavien P. HOPE for human liver grafts obtained from donors after cardiac death. J Hepatol 2014 Apr;60(4):765-772. [doi: 10.1016/j.jhep.2013.11.023] [Medline: 24295869]

14. Saat TC, van den Akker EK, IJzermans JNM, Dor FJMF, de Bruin RWF. Improving the outcome of kidney transplantation by ameliorating renal ischemia reperfusion injury: lost in translation? J Transl Med 2016 Jan 20;14(1):20 [FREE Full text] [doi: 10.1186/s12967-016-0767-2] [Medline: 26791565]

15. Schlegel A, Kron P, Dutkowski P. Hypothermic machine perfusion in liver transplantation. Current Opinion in Organ Transplantation 2016;21(3):308-314. [doi: 10.1097/mot.0000000000000303]

16. Kron P, Schlegel A, Muller X, Gaspert A, Clavien P, Dutkowski P. Hypothermic Oxygenated Perfusion: A Simple and Effective Method to Modulate the Immune Response in Kidney Transplantation. Transplantation 2019;103(5):e128-e136. [doi: 10.1097/tp.0000000000002634]

17. Pascual J, Zamora J, Pirsch JD. A systematic review of kidney transplantation from expanded criteria donors. Am J Kidney Dis 2008 Sep;52(3):553-586. [doi: 10.1053/j.ajkd.2008.06.005] [Medline: 18725015]

18. Dutkowski P, Polak WG, Muiesan P, Schlegel A, Verhoeven CJ, Scalera I, et al. First Comparison of Hypothermic Oxygenated PErfusion Versus Static Cold Storage of Human Donation After Cardiac Death Liver Transplants. Annals of Surgery 2015;262(5):764-771. [doi: 10.1097/sla.0000000000001473]

19. Jochmans I, Moers C, Smits JM, Leuvenink HGD, Treckmann J, Paul A, et al. Machine Perfusion Versus Cold Storage for the Preservation of Kidneys Donated After Cardiac Death. Annals of Surgery 2010;252(5):756-764. [doi: 10.1097/sla.0b013e3181ffc256]

20. Charlson ME, Pompei P, Ales KL, MacKenzie C. A new method of classifying prognostic comorbidity in longitudinal studies: Development and validation. Journal of Chronic Diseases 1987 Jan;40(5):373-383. [doi:

10.1016/0021-9681(87)90171-8]

21. Jupiter DC. Propensity Score Matching: Retrospective Randomization? J Foot Ankle Surg 2017 Mar;56(2):417-420. [doi: 10.1053/j.jfas.2017.01.013] [Medline: 28231973]

22. Slankamenac K, Graf R, Barkun J, Puhan MA, Clavien P. The Comprehensive Complication Index: A Novel Continuous Scale to Measure Surgical Morbidity. Annals of Surgery 2013;258(1):1-7. [doi: 10.1097/sla.0b013e318296c732]

23. Ravaioli M, De Pace V, Comai G, Busutti M, Del Gaudio M, Amaduzzi A, et al. Successful Dual Kidney Transplantation After Hypothermic Oxygenated Perfusion of Discarded Human Kidneys. Am J Case Rep 2017 Sep 20;18:1009-1013 [FREE Full text] [doi: 10.12659/ajcr.905377] [Medline: 28928357] 
24. Requião-Moura LR, Durão Junior MDS, Matos ACCD, Pacheco-Silva A. Ischemia and reperfusion injury in renal transplantation: hemodynamic and immunological paradigms. Einstein (São Paulo) 2015 Mar;13(1):129-135. [doi: 10.1590/s1679-45082015rw3161]

25. Mancina E, Kalenski J, Paschenda P, Beckers C, Bleilevens C, Boor P, et al. Determination of the preferred conditions for the isolated perfusion of porcine kidneys. Eur Surg Res 2015 Oct 21;54(1-2):44-54. [doi: 10.1159/000366155] [Medline: 25341590]

26. Westerkamp AC, Karimian N, Matton APM, Mahboub P, van Rijn R, Wiersema-Buist J, et al. Oxygenated Hypothermic Machine Perfusion After Static Cold Storage Improves Hepatobiliary Function of Extended Criteria Donor Livers. Transplantation 2016;100(4):825-835. [doi: 10.1097/tp.0000000000001081]

27. Fu Z, Ye Q, Zhang Y, Zhong Z, Xiong Y, Wang Y, et al. Hypothermic Machine Perfusion Reduced Inflammatory Reaction by Downregulating the Expression of Matrix Metalloproteinase 9 in a Reperfusion Model of Donation After Cardiac Death. Artif Organs 2016 Jun 27;40(6):E102-E111. [doi: 10.1111/aor.12658] [Medline: 26813475]

28. Protzel C, Führer A, Hakenberg OW. Terminal renal insufficiency and indication for kidney transplantation [in German]. Urologe A 2015 Oct;54(10):1356-1361. [doi: 10.1007/s00120-015-3906-0] [Medline: 26459577]

29. Boor P, Floege J. Renal allograft fibrosis: biology and therapeutic targets. Am J Transplant 2015 Apr;15(4):863-886 [FREE Full text] [doi: 10.1111/ajt.13180] [Medline: 25691290]

30. Siedlecki A, Irish W, Brennan DC. Delayed graft function in the kidney transplant. Am J Transplant 2011 Nov;11(11):2279-2296 [FREE Full text] [doi: 10.1111/j.1600-6143.2011.03754.x] [Medline: 21929642]

31. Kayler L, Srinivas TR, Schold JD. Influence of CIT-induced DGF on kidney transplant outcomes. Am J Transplant 2011 Dec;11(12):2657-2664 [FREE Full text] [doi: 10.1111/j.1600-6143.2011.03817.x] [Medline: 22051325]

\author{
Abbreviations \\ ATP: adenosine triphosphate \\ CIT: cold ischemic time \\ CRF: case report file \\ DGF: delayed graft function \\ ECD: extended criteria donor \\ eGFR: estimated glomerular filtration rate \\ ELISA: enzyme-linked immunosorbent assay \\ ESRD: end-stage renal disease \\ HOPE: hypothermic oxygenated machine perfusion \\ HTK: histidine-tryptophan-ketoglutarate \\ ICH-GCP: International Conference on Harmonization-Good Clinical Practice \\ IRI: ischemia-reperfusion Injury \\ kPa: kilopascal \\ MPS: machine perfusion solution \\ NGAL: neutrophil gelatinase-associated lipocalin \\ O2: oxygen \\ pO2: partial pressure of oxygen \\ RWTH: Rheinisch-Westfälische Technische Hochschule
}

COMPARE: cold oxygenated machine preservation of aged renal donation after cardiovascular death transplants

COPE-POMP: preimplantation oxygenated hypothermic machine perfusion reconditioning after cold storage

Edited by G Eysenbach; submitted 09.05.19; peer-reviewed by F Becker, D Eckhoff; comments to author 10.08.19; revised version
received 29.08.19; accepted 30.08.19; published 14.10.19
Please cite as:
Meister FA, Czigany Z, Bednarsch J, Böcker J, Amygdalos I, Morales Santana DA, Rietzler K, Moeller M, Tolba R, Boor P, Rohlfs
W, Neumann UP, Lurje G
Hypothermic Oxygenated Machine Perfusion of Extended Criteria Kidney Allografts from Brain Dead Donors: Protocol for a
Prospective Pilot Study
JMIR Res Protoc $2019 ; 8(10):$ e14622
URL: $\underline{\text { https://www.researchprotocols.org/2019/10/e14622 }}$
doi: $\underline{10.2196 / 14622}$
PMID: $\underline{31613224}$ 
CFranziska Alexandra Meister, Zoltan Czigany, Jan Bednarsch, Jörg Böcker, Iakovos Amygdalos, Daniel Antonio Morales Santana, Katharina Rietzler, Marcus Moeller, René Tolba, Peter Boor, Wilko Rohlfs, Ulf Peter Neumann, Georg Lurje. Originally published in JMIR Research Protocols (http://www.researchprotocols.org), 14.10.2019. This is an open-access article distributed under the terms of the Creative Commons Attribution License (https://creativecommons.org/licenses/by/4.0/), which permits unrestricted use, distribution, and reproduction in any medium, provided the original work, first published in JMIR Research Protocols, is properly cited. The complete bibliographic information, a link to the original publication on http://www.researchprotocols.org, as well as this copyright and license information must be included. 